\title{
The novel 19q13 KRAB zinc-finger tumour suppressor ZNF382 is frequently methylated in oesophageal squamous cell carcinoma and antagonises Wnt/ $\beta$-catenin signalling
}

\author{
Chong Zhang ${ }^{1}$, Tingxiu Xiang ${ }^{1}$, Shuman Li ${ }^{2}$ Lin Ye ${ }^{1}$, Yixiao Feng ${ }^{1}$, Lijiao Pei ${ }^{1}$, Lili Li ${ }^{3}$, Xiangyu Wang ${ }^{3}$, Ran Sun , \\ Guosheng Ren ${ }^{1}$ and Qian Tao ${ }^{1,3}$
}

\begin{abstract}
Zinc finger proteins (ZFPs) are the largest transcription factor family in mammals. About one-third of ZFPs are Krüppelassociated box domain (KRAB)-ZFPs and involved in the regulation of cell differentiation/proliferation/apoptosis and neoplastic transformation. We recently identified ZNF382 as a novel KRAB-ZFP epigenetically inactivated in multiple cancers due to frequent promoter CpG methylation. However, its epigenetic alterations, biological functions/ mechanism and clinical significance in oesophageal squamous cell carcinoma (ESCC) are still unknown. Here, we demonstrate that ZNF382 expression was suppressed in ESCC due to aberrant promoter methylation, but highly expressed in normal oesophagus tissues. ZNF382 promoter methylation is correlated with ESCC differentiation levels. Restoration of ZNF382 expression in silenced ESCC cells suppressed tumour cell proliferation and metastasis through inducing cell apoptosis. Importantly, ZNF382 suppressed Wnt/ $\beta$-catenin signalling and downstream target gene expression, likely through binding directly to FZD1 and DVL2 promoters. In summary, our findings demonstrate that ZNF382 functions as a bona fide tumour suppressor inhibiting ESCC pathogenesis through inhibiting the Wnt/ $\beta$ catenin signalling pathway.
\end{abstract}

\section{Introduction}

Oesophageal cancer is the eighth most common cancer with the sixth highest cancer mortality rate worldwide and a very low 5 -year survival of $15 \%-25 \%^{1,2}$. Oesophageal squamous cell carcinoma (ESCC) comprises $90 \%$ of oesophageal cancer as the predominant type in China; the

\footnotetext{
Correspondence: Guosheng. Ren (rgs726@163.com)

Qian Tao (qtao@cuhk.edu.hk)

${ }^{1}$ Chongqing Key Laboratory of Molecular Oncology and Epigenetics, The First Affiliated Hospital of Chongqing Medical University, Chongqing, China

${ }^{2}$ Department of Oncology, The Second Affiliated Hospital of Chongqing Medical University, Chongqing, China

Full list of author information is available at the end of the article.

These authors are co-first authors: Chong Zhang, Tingxiu Xiang.

Edited by A. Oberst
}

remaining cases are oesophageal adenocarcinoma $(\mathrm{EAC})^{1,3}$. The incidence of oesophageal cancer varies geographically with the highest in a belt extending through central Asian to North-Central China ${ }^{1,2,4,5}$, reaching an incidence of $>100 / 100,000$ population annually ${ }^{2,6,7}$. The major risk factors for ESCC are poor nutrition, tobacco and alcohol consumption, whereas diet, obesity and gastroesophageal reflux disease are also EAC risk factors ${ }^{4,8,9}$. The incidence of ESCC has been decreasing a bit, but its 5-year survival remains poor; therefore a better understanding of ESCC pathogenesis is urgently needed ${ }^{3,6,10-13}$. Substantial evidences have shown that epigenetic modifications, primarily aberrant promoter CpG methylation of tumour suppressor

\section{(c) The Author(s) 2018}

(c) (i) Open Access This article is licensed under a Creative Commons Attribution 4.0 International License, which permits use, sharing, adaptation, distribution and reproduction in any medium or format, as long as you give appropriate credit to the original author(s) and the source, provide a link to the Creative Commons license, and indicate changes were made. The images or other third party material in this article are included in the article's Creative Commons license, unless indicated otherwise in a credit line to the material. If material is not included in the article's Creative Commons license and your intended use is not permitted by statutory regulation or exceeds the permitted use, you will need to obtain permission directly from the copyright holder. To view a copy of this license, visit http://creativecommons.org/licenses/by/4.0/. 
genes (TSGs), consistently contribute to malignant transformation ${ }^{4,14,15}$. Alterations of promoter hypermethylation occur frequently in ESCC and involve multiple genes required for ESCC carcinogenesis ${ }^{14,16}$. Therefore, it is crucial to investigate the epigenetic abnormalities in ESCC.

Zinc finger proteins (ZFPs) comprise the largest group of transcription factors. Their zinc finger domains bind to gene promoters to activate or repress gene expres$\operatorname{sion}^{17}$. Nearly one-third of mammalian ZFPs contain a highly conserved Krüppel-associated box (KRAB) motif, which contributes to transcriptional repression by recruitment of histone deacetylase (HDAC) complexes $^{18-21}$. We recently identified a novel ZFP ZNF382, the human homologue of rat tumour suppressor KS1 located at $19 \mathrm{q} 13.12$, that functions as a transcriptional repressor by binding to the KAP-1 co-repressor protein $^{22}$. ZNF382 is widely expressed in normal tissues but reduced or silenced in multiple carcinomas due to aberrant promoter $\mathrm{CpG}$ methylation ${ }^{22-24}$. Although evidence has indicated that ectopic expression of ZNF382 suppresses tumour cell proliferation and promotes apoptosis, its biological functions and underlying mechanisms in ESCC pathogenesis remain to be investigated $^{24}$.

Wnt signalling includes canonical Wnt/ $\beta$-catenin pathway and noncanonical pathway and is an oncogenic activation event in many cancers, especially in gastrointestinal cancers. Numerous studies have reported that Wnt signalling is associated with the initiation and progression of human ESCC. Furthermore, comprehensive genomic analysis of ESCC revealed that altered genes in Wnt pathway were identified in $\sim 86 \%$ of ESCC cases, adding to our understanding of pathogenenic role of Wnt pathway in ESCC tumorigenesis ${ }^{25,26}$. In this study, we investigated the methylation status of ZNF382 in primary ESCC and its biological functions in silenced ESCC cell lines. We further explored the mechanism of ZNF382 on tumour suppression of ESCC.

\section{Results}

Promoter methylation leads to ZNF382 downregulation in ESCC

ZNF382 downregulation in some carcinomas has been previously reported ${ }^{24}$. We thus further analysed ZNF382 mRNA expression via qRT-PCR in 15 cases of ESCC and paired adjacent non-cancerous tissues, and we found that ZNF382 expression in the ESCC samples was significantly reduced compared with paired adjacent non-cancerous tissues (Fig. 1a). We then evaluated ZNF382 expression in a panel of ESCC cell lines and normal oesophagus tissues by real-time PCR (Fig. 1b) and qRT-PCR (Supplemental Fig. 1). ZNF382 was highly expressed in normal oesophagus tissues, but almost completely silenced in
KYSE150, KYSE410 and KYSE510 cells. We also examined ZNF382 protein expression in ESCC and paired adjacent non-cancer tissues by immunohistochemistry (IHC). ZNF382 protein expression was significantly weaker in ESCC than in adjacent non-cancerous tissues and primarily localised in cell nuclei (Fig. 1c). In addition, ZNF382 expression was analysed using the online Gene Expression across Normal and Tumour (GENT) tissue database (http://medical-genome.kribb.re.kr/GENT/ search/search.php), and ZNF382 was also found to be downregulated in ESCC tissues compared with normal oesophageal epithelial tissues (Fig. $1 \mathrm{~d}, p=0.0016$ ). Furthermore, the correlation of ZNF382 expression and overall survival (OS) in oesophageal cancer patients was investigated according to the cBioPortal for Cancer Genomics (http://www.cbioportal.org/) in The Cancer Genome Atlas (TCGA) database. Higher levels of ZNF382 expression were associated with a better OS rate in oesophageal cancer patients (Fig. 1e, $p=0.028$ ), suggesting that ZNF382 may be an independent prognostic factor in oesophageal cancer.

Abnormal promoter methylation is a critical mechanism responsible for the silencing of TSGs in tumourigenesis $^{27}$. Our previous research demonstrated that ZNF382 is frequently methylated in solid cancers. To observe whether ZNF382 expression is regulated by promoter methylation, we treated KYSE150, KYSE410 and KYSE510 cells with the demethylation drug Aza combined with the HDAC inhibitor trichostatin A (TSA), and the synergistic treatments significantly restored ZNF382 expression, together with increased unmethylated alleles and decreased methylated alleles (Fig. 1f). In addition, ZNF382 promoter methylation in primary oesophageal cancer tissues and normal oesophagus tissues from a human disease methylation database (http://www. biobigdata.com/diseasemeth/) was analysed. ZNF382 methylation was far more prevalent in oesophageal cancer tissues than in normal oesophagus tissues (Fig. 1g, $p=$ 0.0023). Thus, the overall results indicate that promoter methylation may be a primary mechanism of ZNF382 silencing in ESCC cell lines.

\section{ZNF382 methylation in ESCC is correlated with clinicopathological features}

To further investigate ZNF382 promoter methylation status in ESCC, methylation-specific PCR (MSP) was performed in primary ESCC and the normal oesophagus tissues. The data showed that the ZNF382 promoter was hypermethylated in 87\% (99/114) of ESCC tissues and in 33\% (1/3) of normal oesophageal tissues, suggesting the frequent methylation of ZNF382 in ESCC (Fig. 1h-i and Table 1). Next, we analysed the relationship between ZNF382 methylation status and clinical features, and a significant association was found with tumour differentiation (Table 2) ( $\chi^{2}$ test, $p=0.029$ ). 


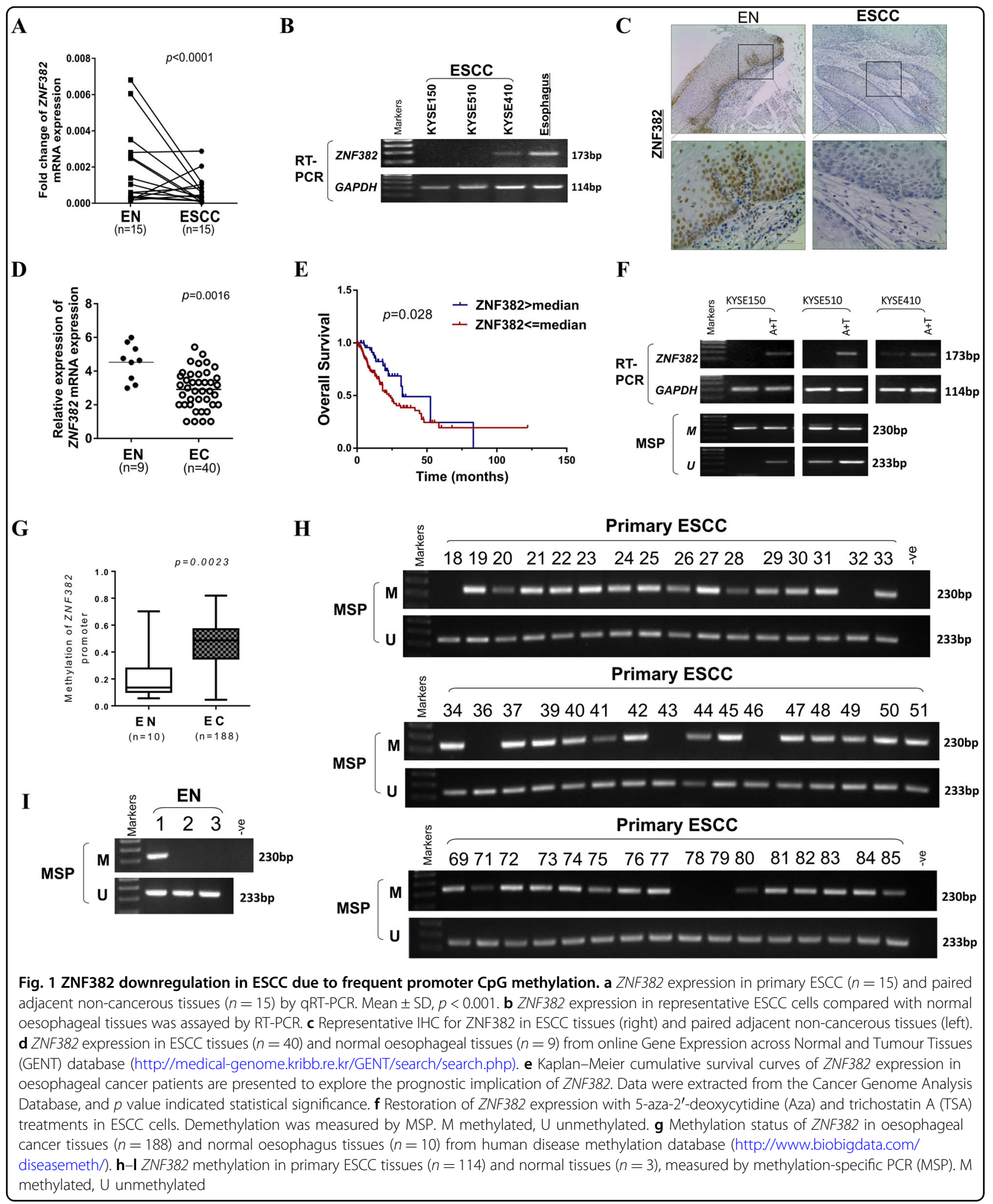


Table 1 The promoter methylation of ZNF382 in primary ESCC

\begin{tabular}{llll}
\hline Samples & \multicolumn{2}{l}{ ZNF382 methylation status } & $\begin{array}{l}\text { Frequency } \\
\text { ofmethylation }\end{array}$ \\
\cline { 2 - 3 } & Methylated & Unmethylated & \\
\hline ESCC $(n=$ & 99 & 15 & $87 \%(99 / 114)$ \\
$114)$ & & & $33 \%(1 / 3)$ \\
EN $(n=3)$ & 1 & 2 & $75 \%(12 / 16)$ \\
Adjacent & 12 & 4 & \\
tissues $(n=16)$ & & & \\
\hline
\end{tabular}

ESCC Oesophageal squamous cell carcinoma, EN Normal oesophagus tissues

\section{Ectopic expression of ZNF382 suppresses ESCC cell proliferation}

ZNF382 ectopic expression in ZNF382-stably transfected KYSE150, KYSE410 and KYSE510 cells was verified by RT-PCR and western blot, respectively (Fig. 2a, b). To investigate the effect of ZNF382 on ESCC cell proliferation, cell viability and colony formation assays were performed. ZNF382-transfected cells exhibited lower viability at 24,48 and $72 \mathrm{~h} \mathrm{(Fig.} \mathrm{2c)} \mathrm{and} 60 \%$ to $80 \%$ less colony formation than controls (Fig. 2d). In addition, we also carried out Edu cell proliferation assays, and a significant decrease in the percentage of Edu-positive cells was observed after transfection of ESCC cells with ZNF382 (Fig. 2e and Supplemental Figure 2).

\section{ZNF382 induces G2/M cell cycle arrest and apoptosis in ESCC cells}

A cell cycle assay was performed to determine how ZNF382 affects cell proliferation. Compared with controls, ZNF382 significantly increased KYSE150, KYSE410 and KYSE510 cells in the G2/M phase after ZNF382 transfections (Fig. 2f). This effect was accompanied by Cdc25C, Cdc2 and CyclinB1 inhibitions (Fig. 2g). Flow cytometry analysis of cell apoptosis was performed to evaluate the mechanisms of ZNF382 suppression of tumour cell growth. As shown in Figure $2 \mathrm{~h}$, the percentage of apoptotic cells was remarkably increased after ZNF382- transfection in ESCC cells. Caspases were involved in the proteolytic cascade, which further induced apoptosis. Western blots showed that ZNF382 significantly increased the cleavage of caspase 8 , caspase 3 and PARP in ESCC cells compared with vector controls (Fig. 2i).

\section{ZNF382 inhibits ESCC cell migration and invasion}

To investigate the effect of ZNF382 on cell migration, wound healing assays were carried out. ZNF382-stably transfected ESCC cells migrated into the wounded areas slower than the control cells at $24 \mathrm{~h}$ (Fig. 3a). Moreover, the effect of ZNF382 on cell migration and invasion were further analysed by transwell assay. The results showed that ectopic expression of ZNF382 significantly decreased the number of migrating (Fig. 3b, c) and invading cells (Fig. 3d, e) compared with controls. The effect of ZNF382 on ESCC cell metastasis was mediated by E-cadherin (CDH1) upregulation and vimentin (VIM), N-cadherin (CDH2), SNAIL1 and SLUG downregulation (Fig. 3f).

\section{Knockdown of ZNF382 promotes cell growth and induces metastasis}

To further validate the suppression function of ZNF382 in ESCC, we investigated the effect of ZNF382 through knockdown of ZNF382 in ZNF382-expressing ESCC cell line EC1 by siRNA transfection. Knockdown efficiency was detected by western blot (Fig. 4a). Knockdown of ZNF382 markedly increased EC1 cell viability, according to CCK8 assays compared with cells transfected with control siRNA (Fig. 4b). To evaluate the effect of ZNF382 on cell cycle regulation, flow cytometry assays were employed. The distribution of EC1 cells in G2/M phase was significantly reduced after knocking down ZNF382 (Fig. 4c). Moreover, we observed enhanced cell migration and invasion after ZNF382 knocking down. Compared with control, the number of migrated and invading EC1 cells were dramatically increased after knockdown of ZNF382 (Fig. 4d). These knockdown data confirmed that ZNF382 functions as a tumour suppressor in ESCC cells.

\section{Identification of differentially expressed genes regulated by ZNF382 in ESCC}

To better investigate the underlying mechanism of ZNF382 in ESCC, RNA-Seq was performed in ZNF382stably transfected KYSE150 cells. The entire distribution of differentially expressed genes was shown by a volcano plot, and a total of 3276 upregulated and 3557 downregulated genes were observed (Fig. $5 \mathrm{a}, " p<0.05$ ). We next performed the hierarchical cluster analysis of 42 differentially expressed genes selected on the basis of their expression patterns, and two major groups were classified (Fig. 5b). To further confirm the differential expression of genes identified by RNA-Seq analysis, qRT-PCR was performed to validate the gene expression levels, and 15 differentially expressed genes modulated by ZNF382 were verified in both KYSE150 and KYSE410 cells (Fig. 5c-e).

Moreover, these significantly regulated genes identified in both KYSE150 and KYSE410 cells were involved in cell metastasis (SLUG, SNAIL and SMAD3), cell growth (CSF1 and $V E G F A$ ), stress-induced protector (SESN3) and stem cell regulation (SOX2 and $S T A T 5 B$ ), adding to our understanding of the possible mechanisms of ZNF382 tumour suppression in ESCC. 
Table 2 The correlation between ZNF382 promoter methylation and clinicopathological feature in ESCC

\begin{tabular}{|c|c|c|c|c|}
\hline \multirow[t]{2}{*}{ Clinicopathological features } & \multirow{2}{*}{$\begin{array}{l}\text { Numbers } \\
N=(114)\end{array}$} & \multicolumn{3}{|c|}{ ZNF382 methylation status } \\
\hline & & Methylated & Unmethylated & $p$ value \\
\hline Age & & & & 0.422 \\
\hline$\leq 60$ & 35 & 30 & 5 & \\
\hline$>60$ & 79 & 68 & 11 & \\
\hline Gender & & & & 0.366 \\
\hline Male & 92 & 78 & 14 & \\
\hline Female & 22 & 20 & 2 & \\
\hline Tumour Location & & & & 0.411 \\
\hline Upper & 17 & 14 & 3 & \\
\hline Middle & 56 & 47 & 9 & \\
\hline Lower & 40 & 37 & 3 & \\
\hline Unknown & 1 & 0 & 1 & \\
\hline Differentiation & & & & 0.029 \\
\hline Moderately/Well & 89 & 80 & 9 & \\
\hline Poorly & 25 & 18 & 7 & \\
\hline Tumour Grade & & & & 0.076 \\
\hline $\mathrm{T} 1-2$ & 34 & 30 & 4 & \\
\hline T3-4 & 79 & 68 & 11 & \\
\hline Unknown & 1 & 0 & 1 & \\
\hline Tumour Size & & & & 0.465 \\
\hline$>5.0 \mathrm{~cm}$ & 19 & 17 & 2 & \\
\hline$\geq 2.0 \mathrm{~cm} \leq 5.0 \mathrm{~cm}$ & 75 & 67 & 8 & \\
\hline$<2.0 \mathrm{~cm}$ & 14 & 12 & 2 & \\
\hline Unknown & 6 & 4 & 2 & \\
\hline Tumour Stage & & & & 0.089 \\
\hline$|/| \mid$ & 70 & 61 & 9 & \\
\hline III/IV & 43 & 37 & 6 & \\
\hline Unknown & 1 & 1 & 0 & \\
\hline Lymph Nodes Metastasis & & & & 0.356 \\
\hline Positive & 40 & 34 & 6 & \\
\hline Negative & 74 & 65 & 9 & \\
\hline Distant Metastasis & & & & 0.336 \\
\hline Positive & 1 & 1 & 0 & \\
\hline Negative & 113 & 97 & 16 & \\
\hline
\end{tabular}

The presence of italics values means whether there is a significant association between ZNF382 methylation and the feature. As p<0.05 was set to be statistically significant, so $p=0.029$ means there's statistically significant association between ZNF382 methylation and tumor differentiation

ZNF382 inhibits Wnt/ $\beta$-catenin signalling through binding to DVL2 and FZD1 promoters

ChIP-Seq data of HEK293 cells stably expressing eGFPZNF382 fusion protein were downloaded from the Gene
Expression Omnibus (GEO) database. Enriched peaks located in the transcriptional start sites of FZD1 and $D V L 2$ genes were obtained, suggesting that FZD1 and DVL2 are the direct targets of ZNF382 (Supplemental 


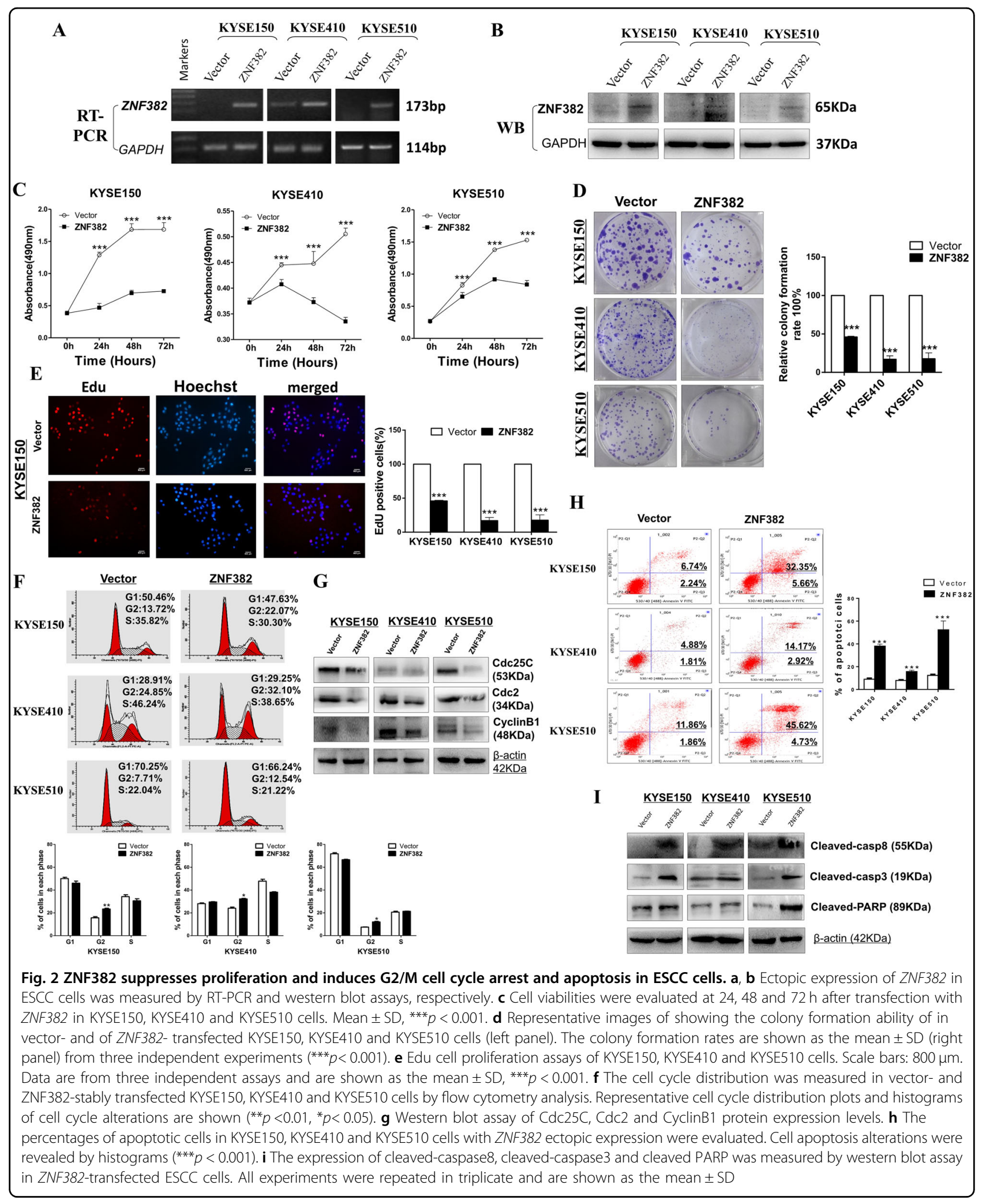




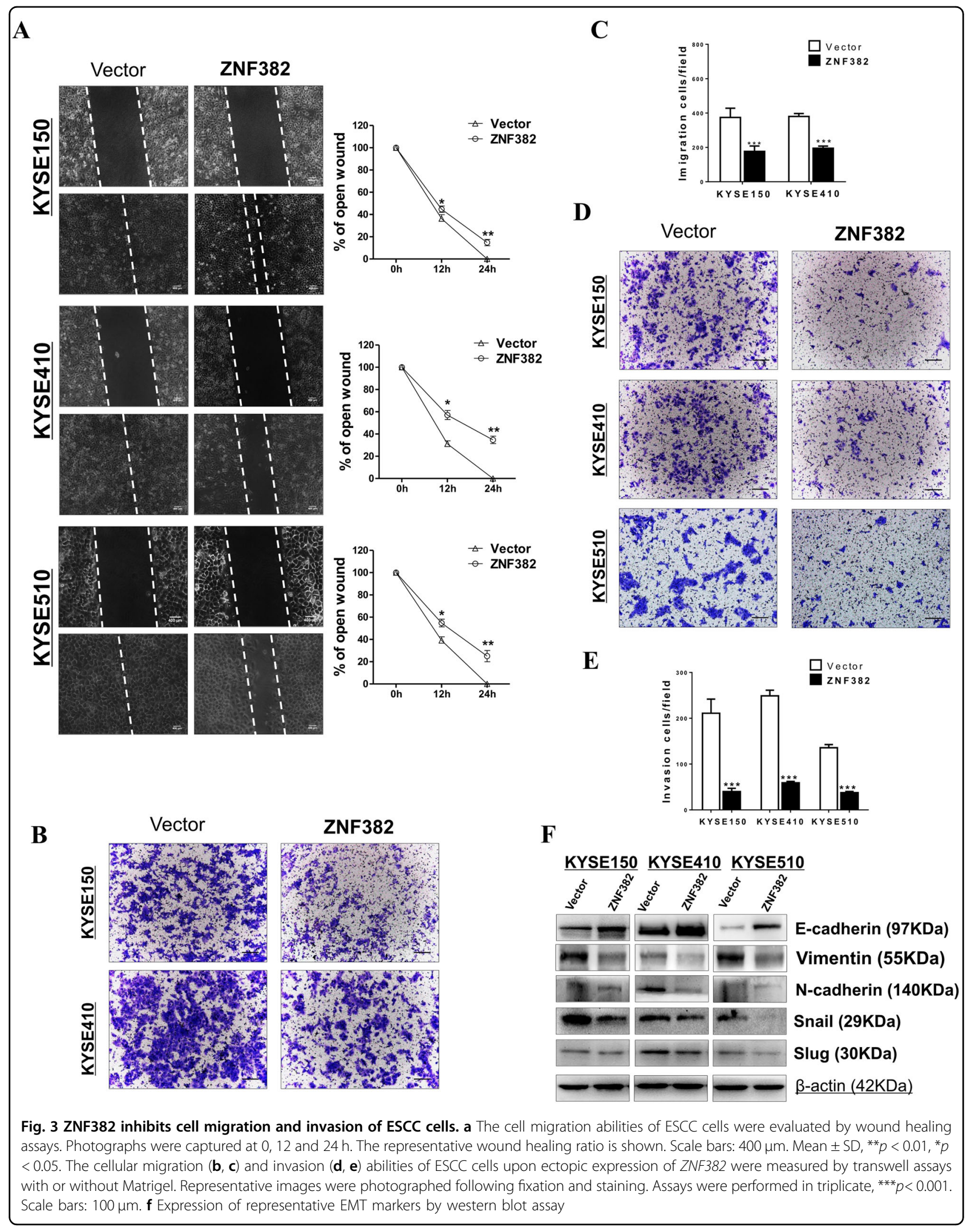


A

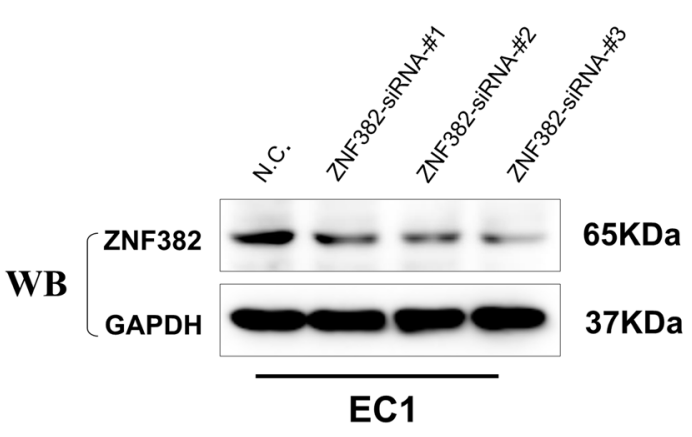

$\mathbf{C}$

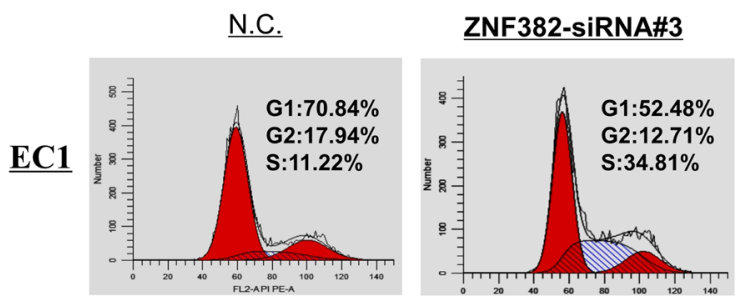

B

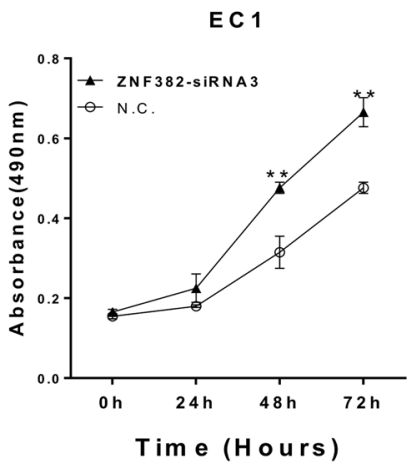

D
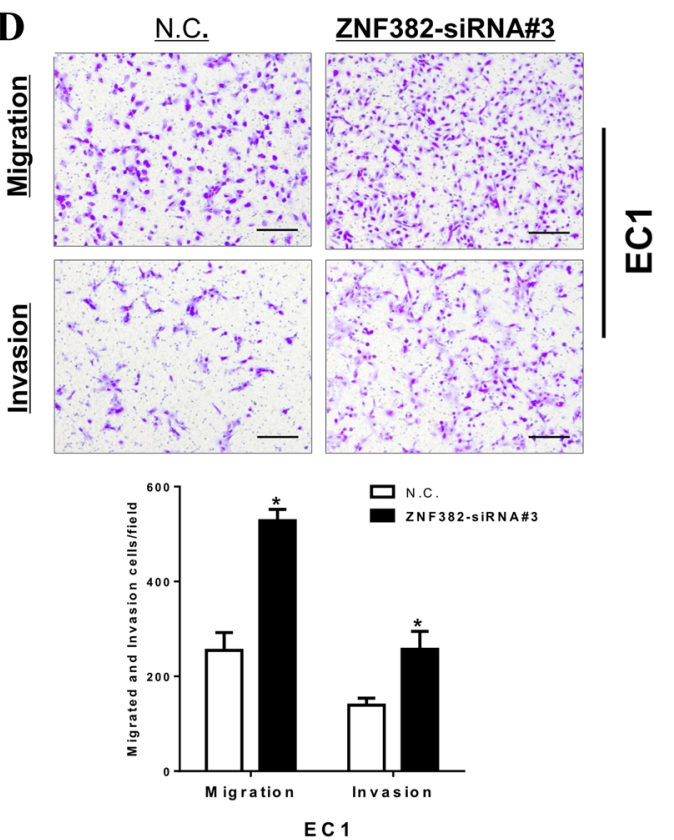

Fig. 4 Knockdown of ZNF382 promotes ESCC cell growth and induces metastasis. a Evaluation of ZNF382 knockdown in EC1 cells after transfection with ZNF382 siRNA and si-Scramble by western blot. b Knockdown of ZNF382 on cell viability was measured by cell viability assay. Each experiment was repeated three times. ${ }^{* *} p<0.01$. c Cell cycle distribution after ZNF382 knockdown in EC1 cells. Each experiment was repeated three times. ${ }^{* *} p<0.01$. $\mathbf{d}$ Transwell assay showed cell migration and invasion of EC1 cells after ZNF382 knockdown. Scale bars: $200 \mu$ m. Each experiment was repeated three times. ${ }^{*} p<0.05$

Fig. 3). List of Chip-Seq peaks for ZNF382 targets were listed in Table S2.

Thus, we hypothesised that ZNF382 likely plays a role in Wnt/ $\beta$-catenin signalling through directly binding to FZD1 and DVL2 promoters. Reduced FZD1 and DVL2 accompanied by downstream target genes such as $C$ $M Y C, C C N D 1, M M P 2$ and $M M P 3$ expression levels were observed at both the transcriptional (Fig. $5 \mathrm{f}-\mathrm{g}$ ) and protein levels (Fig. 5h). Then, we examined the expression of active $\beta$-catenin via western blot. As shown in Figure $5 \mathrm{~h}$, the expression of total $\beta$-catenin remained unchanged, while the expression of active $\beta$-catenin decreased. In addition, inhibition of TOPflash luciferase activity more fully demonstrated that ZNF382 antagonised Wnt/ $\beta$ catenin signalling by decreasing active $\beta$-catenin levels (Fig. 5i). These results conclusively suggest that ZNF382 exerts tumour suppression function through antagonising Wnt/ $\beta$-catenin signalling by directly binding to $D V L 2$ and FZD1 promoters.

\section{Pathway enrichments and protein-protein interaction network}

Gene Ontology (GO) terms and Kyoto Encyclopedia of Genes and Genomes (KEGG) pathway enrichments based on RNA-Seq data were further analysed and are shown in Figure $6 \mathrm{a}, \mathrm{b}$. The Wnt pathway was significantly enriched 


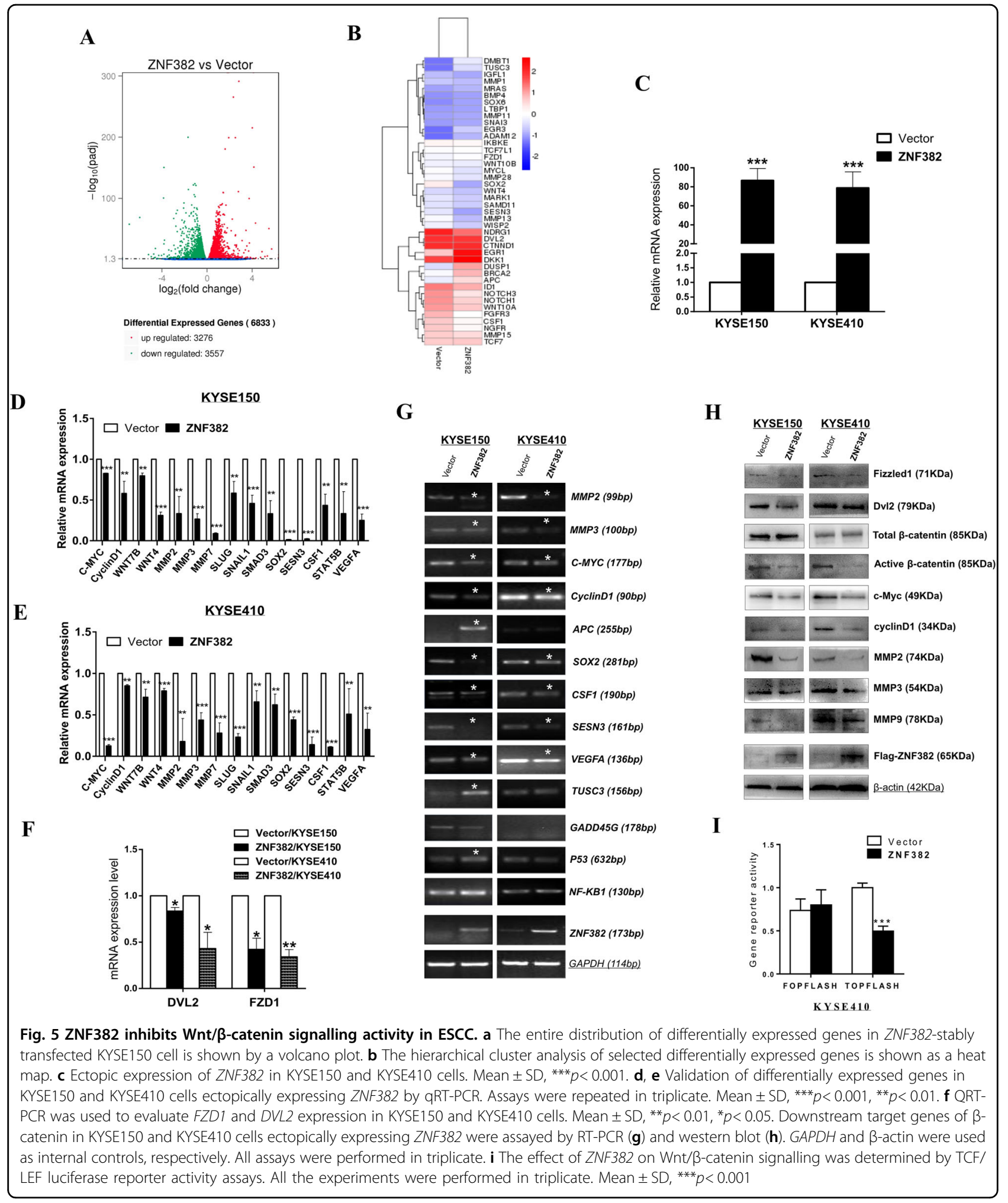

in both GO and KEGG pathways and other processes, such as apoptosis induction, negative regulation of RNA polymerase II transcription and regulation of stem cell pluripotency were also included. In addition, the protein-protein interaction (PPI) network showed interactions within the network of genes transcribed by ZNF382, which involved cell development, cell cycle regulation and metastasis (Fig. 6c). 


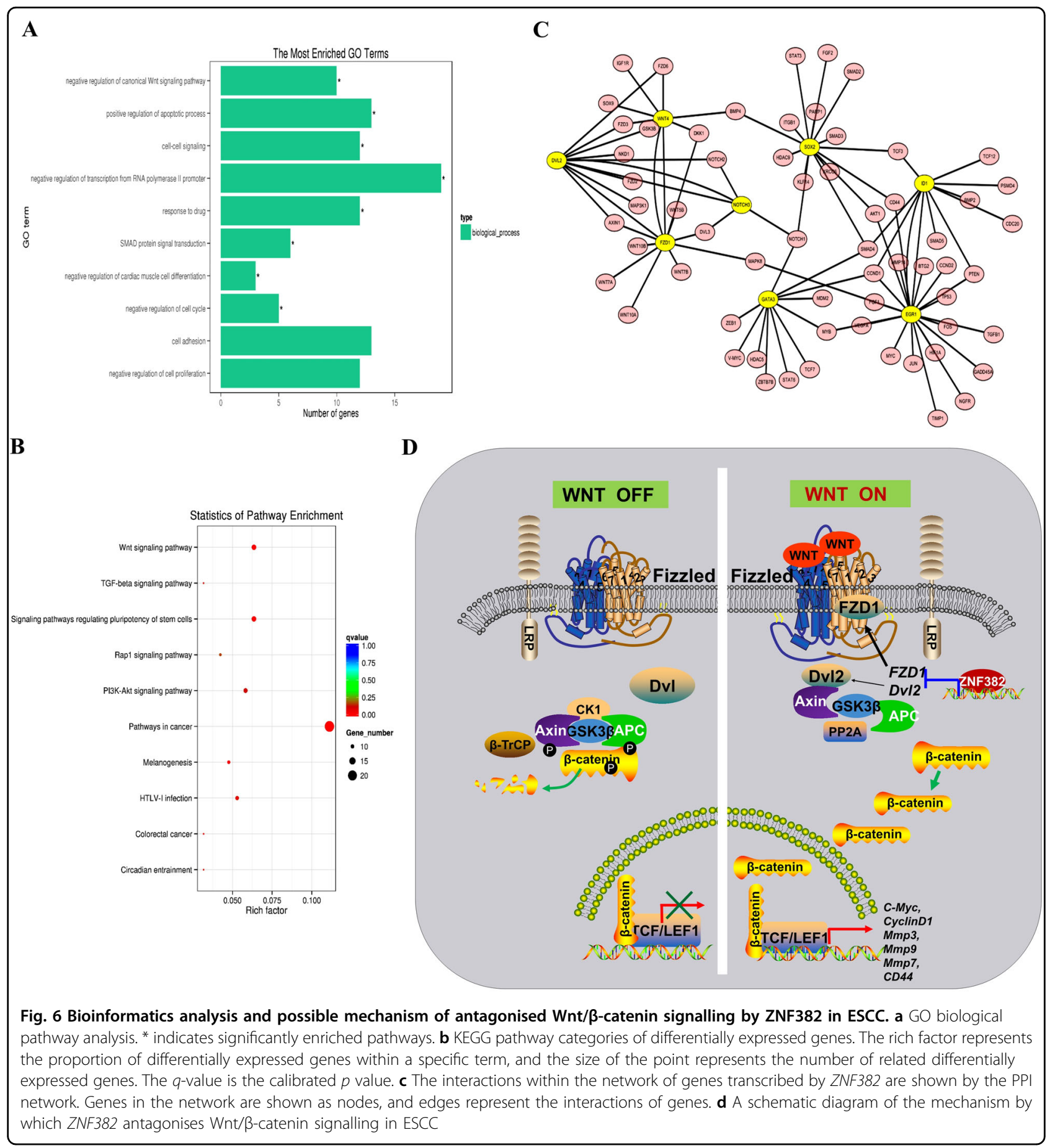

\section{Discussion}

ZFPs comprising 742 members and involving 423 genes make up the largest transcription factor family in mammals ${ }^{28,29}$. The KRAB domain, located in the amino terminal of ZFP, exerts transcriptional repressor functions ${ }^{16,19,21,30}$ by sequence-specific DNA methylation $^{29,31,32}$. Due to the potent repressor domain, ZFPs play an essential role in cell metabolism, differentiation and apoptosis $^{21,33,34}$. We investigated the novel 19q13 KRABZFP ZNF382, which is orthologous to rat KS1, and found that ZNF382 was highly expressed in normal tissues but frequently silenced in multiple carcinomas due to promoter CpG methylation ${ }^{22,24,29,35}$. ZNF382 was significantly reduced or silenced in ESCC tissues compared with normal oesophageal tissues. The MSP assay also revealed that ZNF382 promoter was methylated in $87 \%$ 
(99/114) of ESCC cases, suggesting a high frequency of ZNF382 promoter methylation in ESCC. In addition, data from TCGA database showed that higher level of ZNF382 heralded a better OS in ESCC patients, indicating that ZNF382 might be an independent prognostic factor for ESCC patients.

Here, we demonstrated the tumour suppressive effects of ZNF382 in ESCC cells. Our results indicate that inhibition of cell proliferation by ectopic expression of ZNF382 in ESCC cells might be through G2/M cell cycle arrest and apoptosis, accompanied by reduced of Cdc25C, $\mathrm{Cdc} 2$ and CyclinB1 checkpoint regulators and induced cleavage of apoptotic markers caspase8, caspase3 and PARP. The effect of ZNF382 on cell metastasis was further evaluated by scratch and transwell assays. The decreased migration and invasion abilities of ESCC cells, with upregulation of epithelial marker $\mathrm{CDH} 1$ and downregulation of VIM, CDH2, SNAIL1 and SLUG resulted from negative regulation by ZNF382, which was also reflected in the epithelial-mesenchymal transition (EMT) of ESCC cells.

RNA-Seq was additionally performed to explore the underlying mechanism of ZNF382 in ESCC, and a total of 3276 upregulated and 3557 downregulated genes were identified. Subsequently, 42 differentially expressed genes were selected among which 29 DEGs exhibited a fold change $>1.5$, for further hierarchical cluster analysis. Validation by qRT-PCR revealed 15 differentially expressed genes modulated by ZNF382 that were involved in cell metastasis (SLUG, SNAIL and SMAD3), cell growth (CSF1 and VEGFA), stress-induced protection (SESN3) and stem cell regulation (SOX2 and STAT5B) were observed in both KYSE150 and KYSE410 cells.

Wnt signalling pathway participates in the carcinogenesis of multiple types of tumours, especially gastrointestinal cancers. Numerous articles have reported that Wnt pathway alterations are involved in the development of oesophageal squamous carcinomas. Wnt signalling mainly includes canonical and noncanonical pathways. The canonical pathway (WnT/ $\beta$-catenin signalling) is the most frequently reported pathway and has been associated with the regulation of tumour growth, metastasis, and transformation.

In the present investigation, we identified two target genes, FZD1 and DVL2, from ChIP-Seq of HEK293 cells stably transfected with ZNF382. Both of these genes are critical elements in the Wnt pathway. ZNF382 negatively regulates the $\mathrm{Wnt} / \beta$-catenin signalling by directly binding to $F Z D 1$ and $D V L 2$ promoters, inhibiting $\beta$-catenin activation and $\mathrm{T}$ cell factor/lymphoid-enhancer factor (TCF/ LEF)-dependent luciferase reporter activity, subsequently downregulating the downstream target genes of $\beta$-catenin signalling including $C-M Y C, C Y C L I N D 1, M M P 2, M M P 3$ and $M M P 9$ expressions (Fig. 6d).
It is worth mentioning that amplification of CYCLIND1, an essential downstream target gene of $\beta$-catenin, has been detected in ESCC samples ${ }^{1}$. Overexpression of CYCLIND1 is common in ESCC cell lines and is a poor prognostic marker of poor ESCC outcomes, due to its strong association with increased lymph nodes or distant metastasis, high proliferation rates and poor responses to chemotherapy ${ }^{1}$. Thus, evaluation of CYCLIND1 status is critical for ESCC patients. Here, we found that ZNF382 negatively modulates $\mathrm{Wnt} / \beta$-catenin signalling, further inhibiting target gene CYCLIND1 expression. These findings indirectly illustrate that ZNF382, as a TSG, could be a promising prognostic predictor for ESCC patient outcomes.

\section{Conclusions}

In conclusion, ZNF382 was frequently inactivated by promoter CpG methylation, and suppressed ESCC carcinogenesis through directly regulating $W n t / \beta$-catenin signalling.

\section{Materials and methods}

\section{Primary tumour samples and cell lines}

Multiple ESCC cell lines including KYSE150, KYSE410 and KYSE510 were used ${ }^{24}$. The cells were cultured in RPMI 1640 medium (Gibco-BRL, Karlsruhe, Germany), supplemented with $10 \%$ foetal bovine serum (Gibco-BRL), at $37^{\circ} \mathrm{C}$ in $5 \% \mathrm{CO}_{2}$ atmosphere. Primary ESCC tissues, adjacent non-cancerous tissues and normal oesophageal tissues were obtained from patients who underwent surgery at the Department of Cardiothoracic Surgery of the First Affiliated Hospital of Chongqing Medical University. All samples were stored at $-80^{\circ} \mathrm{C}$ until being evaluated by pathologists at the Chongqing Key Laboratory of Molecular Oncology and Epigenetic of the First Affiliated Hospital of Chongqing Medical University. All participants provided written consent before enrolment, and the study was approved by the Ethics Committees of the First Affiliated Hospital of Chongqing Medical University.

\section{RNA and DNA extraction}

Total RNA was isolated from cells and tissues with the TRIzol reagent (Invitrogen, Carlsbad, CA, USA). Genomic DNA was extracted from cells and tissues with a QIAamp DNA Mini Kit (Qiagen, Hilden, Germany) following the manufacturers' protocols ${ }^{36}$. RNA and DNA concentrations were measured with a NanoDrop 2000 spectrophotometer (Thermo Fisher Scientific, Waltham, MA), and sample quality was determined by gel electrophoresis.

\section{Reverse transcription, semi-quantitative PCR (RT-PCR) and quantitative PCR (qRT-PCR)}

Reverse transcription and semi-quantitative PCR (RTPCR) were performed as previously described using Go- 
Taq polymerase (Promega, Madison, WI) ${ }^{37}$. RT-PCR was performed using a final volume of $10 \mu \mathrm{L}$ reaction mixture containing $2 \mu \mathrm{L}$ cDNA. GAPDH served as an internal control. The amplified PCR products were assayed on $2 \%$ agarose gels. A SYBR Green PCR Master Mix kit (Invitrogen) and an Applied Biosystems 7500 Real-Time PCR System (Applied Biosystems, Foster City, CA) were used for qRT-PCR ${ }^{36}$. The relative expression of ZNF382 was estimated with the $2(-\Delta \mathrm{Ct})$ method $^{38}$ and all assays were performed in triplicate. The primers used are listed in Supplementary Table 1.

\section{5-Aza-2'-deoxycytidine (Aza) and trichostatin A (TSA) treatment}

Combined treatment of Aza, a DNA methyltransferase inhibitor and TSA, a HDAC inhibitor synergistically activated methylated genes. ESCC cell lines were treated with $10 \mathrm{mmol} / \mathrm{L}$ Aza (Sigma-Aldrich, Steinheim, Germany) for three days and $100 \mathrm{nmol} / \mathrm{L}$ TSA (SigmaAldrich) for an additional $24 \mathrm{~h}$ as previously described ${ }^{24}$. Cells were then used for RNA and DNA isolation.

\section{Bisulfite treatment and methylation-specific PCR (MSP)}

To evaluate the methylation status of ZNF382, bisulfite modification of DNA and MSP were performed as previously described ${ }^{24,39,40}$. In brief, bisulfite-treated DNA was amplified by MSP with methylation-specific primers. Methylated and non-methylated DNAs were used as positive and negative controls. MSP products were separated on 2\% agarose gels (MBI Fermentas, Vilnius, Lithuania). The primers are listed in Supplementary Table 1.

\section{Transfection and construction of stable cell lines}

A ZNF382 expression vector (PcDNA3.1-ZNF382FLAG) and a control vector (PcDNA3.1) were used ${ }^{24}$. Plasmids were transfected into ESCC cell lines using Lipofectamine 2000 (Invitrogen) at a final concentration of $4 \mu \mathrm{g}$. The transfected cells were harvested at $48 \mathrm{~h}$. ZNF382-stably transfected ESCC cells were selected with G418 (Invitrogen, Gibco) for 2 weeks.

\section{Knockdown of ZNF382}

ZNF382 siRNA kits were purchased from GenePharma (Jiangsu, China). All transfections were performed using Lipofectamine 2000 (Invitrogen) according to the manufacturer's instruction with a concentration of $50 \mathrm{nM}$ siRNA. The cells were harvested for subsequent assays at $72 \mathrm{~h}$ after transfection.

\section{Cell viability assay}

Stably transfected KYSE150, KYSE410 and KYSE510 cells were seeded in 96-well plates and grown overnight. Cell viabilities were then evaluated with a Cell Counting
Kit-8 (Beyotime Institute of Biotechnology, Jiangsu, China) at 24, 48 and $72 \mathrm{~h}$, respectively. All experiments were independently repeated three times.

\section{Colony formation assay}

Stably transfected KYSE150, KYSE410 and KYSE510 cells were plated in six-well plates and cultured with various concentrations of G418 for 10-14 days. Surviving colonies with $>50$ cells were counted following fixation and stained with gentian violet (Beyotime). All experiments were repeated three times.

\section{5-Ethynyl-2'-deoxyuridine (Edu) cell proliferation assay}

Cell proliferation was evaluated with an Edu cell proliferation kit (RiboBio, Guangzhou, China). Edu is a thymine nucleoside analogue that replaces thymine during DNA replication, and DNA replication activity is assayed by labelling with specific Edu and Apollo fluorescent dyes. All assays were repeated three times.

\section{Wound healing and transwell assay}

Cell mobility was evaluated with a wound healing assay. Briefly, ZNF382-stably transfected KYSE150, KYSE410 and KYSE510 cells were seeded in six-well plates and grown until confluence. Linear scratch wounds were created with a pipette tip and cell migration distance was measured at various times by phase contrast microscopy (Leica DMI4000B, Milton Keynes, Bucks, UK).

Transwell chambers with an $8 \mu \mathrm{m}$ pores (Corning Life Sciences, Bedford, MA) were used with or without Matrigel (BD Biosciences, San Jose, CA) or not to measure cell migration and invasion, respectively. Cells on the lower membrane surface were counted following fixation and staining. All assays were performed in triplicate.

\section{Flow cytometry assay}

Cell cycle arrest and apoptosis were assayed via flow cytometry as previously described ${ }^{37}$. In brief, the cells were harvested with trypsin, fixed with ice-cold $70 \%$ ethanol and stained with propidium iodide (PI) to assay the cell cycle distribution. Apoptosis was assayed with annexin V-fluorescein isothiocyanate and PI following the kit manufacturer's protocol. All data were measured using a Cell Quest kit (BD Biosciences) and experiments were independently repeated in triplicate.

\section{Western blot assay}

Western blot assays were performed as previously described $^{36}$. Briefly, whole cells lysates were prepared with lysis buffer (Beyotime) containing a protease inhibitor cocktail (Sigma-Aldrich, St. Louis, MO). Lysates were sonicated, and the protein concentration was determined using a bicinchoninic acid (BCA) assay. Aliquots of $50 \mu \mathrm{g}$ protein were separated with $10-12 \%$ sodium dodecyl 
sulphate polyacrylamide gel electrophoresis and then transferred to polyvinylidene fluoride membranes (Merck Millipore, Billerica, MA). After blocking with 5\% nonfat milk, the membranes were incubated at $4{ }^{\circ} \mathrm{C}$ overnight with anti-DDK (FLAG) monoclonal antibody (TA50011100; OriGene, Rockville, MD), anti-FLAG M2 antibody (\#14793; Cell Signaling Technology, Danvers, MA), cleaved caspase 3 (\#9661, Cell Signaling Technology), cleaved caspase 8 (\#8592; Cell Signaling Technology), cleaved PARP (\#5625, Cell Signaling Technology), cyclin D1 (\#1677; Epitomics, Burlingame, CA), c-Myc (\#1472-1, Epitomics), active $\beta$-catenin (\#05-665,Merck Millipore), total $\beta$-catenin $(\# 2677$, Cell Signaling Technology), $\beta$ actin (sc-47778; Santa Cruz Biotechnology), E-cadherin (\#1702-1, Epitomics), vimentin (\#2707-1, Epitomics), Ncadherin (ab98952; Abcam), Snail1 (\#3897;Cell Signaling Technology), SLUG (\#9585, Cell Signaling Technology), anti-Frizzled homologue 1 antibody (ab71342, Abcam), Dvl2 (sc-420081, Santa Cruz), Cdc2 (sc-8395, Santa Cruz), Cdc25C (sc-24540, Santa Cruz), or cyclinB1 (sc-4073, Santa Cruz) primary monoclonal antibodies. Membranes were visualised with Electrochemiluminescence Plus Detection Reagents (RPN2132; GE Healthcare Life Science, Buckinghamshire, UK). All assays were independently repeated three times.

\section{Immunohistochemistry (IHC)}

IHC was performed with an UltraSensitive SP Kit (Maixin-Bio, Fujian, China) following the manufacturer's instructions as previously described ${ }^{41,42}$. Cells were incubated with a primary antibody against ZNF382 (1:50 dilution, Sigma-ldrich) overnight at $4{ }^{\circ} \mathrm{C}$ and then incubated with a secondary antibody at $37^{\circ} \mathrm{C}$ for $30 \mathrm{~min}$. All IHC images were captured under a microscope at $\times 400$ magnification.

\section{Dual-luciferase reporter assay}

Briefly, cells were co-transfected with pcDNA3.1ZNF382-FLAG or pcDNA3.1 and TCF-responsive luciferase construct TOPflash or FOPflash reporter plasmids plus Renilla luciferase reporter pRL-TK in 24-well plates. Luciferase activities were read $48 \mathrm{~h}$ later using a dualluciferase reporter assay kit (Promega). All assays were independently performed three times.

\section{RNA sequencing (RNA-Seq)}

RNA-Seq was performed by the Novogene Bioinformatics Technology Company (Beijing, China). RNA integrity was assessed with an RNA Nano 6000 Assay Kit included with the Bioanalyzer 2100 system (Agilent Technologies, CA). Sequencing libraries were prepared using a NEBNext Ultra RNA LibraryPrep Kit for Illumina (New England Biolabs) following the manufacturer's recommendations. RNA-Seq was performed with an
Illumina Hiseq 2000/2500 platform. Bowtie v. $2.0 .6^{43-45}$ and TopHat v. 2.0.9 $9^{46-48}$ were used for reads mapping to the reference genome. Genes with an adjusted $p$ or0.05 and identified with DESeq $^{49,50}$ were considered to be differentially expressed.

\section{Peaks calling for chromatin immunoprecipitation sequencing (ChIP-Seq)}

ChIP-Seq of ZNF382 from human HEK293 cells were obtained from the Gene Expression Omnibus (GEO, https://www.ncbi.nlm.nih.gov/geo) database. The GSM24 24064_ENCFF191YCR_fold_change_over_control_GRCh 38.bigWig file was downloaded. We annotated those peaks by using R package "CHIPseeker" ${ }^{\text {" }}$. Subsequently, the gene region were intercepted with BEDTools ${ }^{52}$ and exported to Integrated Genomics Viewer software to map the peaks ${ }^{53,54}$.

\section{Pathway enrichment analysis}

The function enrichments of differentially expressed genes were analysed with GO classification ${ }^{55}$ and Kyoto Encyclopedia of Genes and Genomes (KEGG) pathways ${ }^{56}$ from the Database for Annotation, Visualization and Integrated Discovery (DAVID, http://david.abcc.ncifcrf. gov/ $/)^{57}$. The level of significance was $p<0.05$.

\section{PPI analysis}

The PPI analysis of target genes was based on the STRING database (https://string-db.org/) and the networks were constructed by Cytoscape ${ }^{58}$.

\section{Statistical analysis}

Data were reported as the means \pm standard deviation (SD). The significance of differences between the experimental and control values were tested the with two-tailed Student's $t$-test. Correlations of methylation status and clinicopathological features were evaluated using the $\chi^{2}$ and Fisher's exact tests. For all tests, $p<0.05$ was considered statistically significant.

\section{Availability of data and materials}

Data of prognostic significance is available from Cancer Genome Atlas database (http://www.cbioportal.org/). CHIP-Seq data analysed during the present study is available from Gene Expression Omnibus database (GEO, https://www.ncbi.nlm.nih.gov/geo). PPI analysis is available from the STRING database (https://string-db.org/). Schematic model drafted with the tool scienceslides software is available at http://www.scienceslides.com/.

\section{Acknowledgements}

This study was supported by National Natural Science Foundation of China (NSFC) (\#81572769, \#31420103915, \#81772869, \#81572327), National Key Research and Development Program of China (\#2017YFE0191700) and the VC special research fund from The Chinese University of Hong Kong. 


\section{Authors' contributions}

C.Z., T.X., and Q.T.: conception and design of the study. C.Z., S.L., L.Y., R.S., Y.F., L. P., L.L., and X.W.: performed experiments and analysed data. C.Z., and L.Y.: collected samples. C.Z.: prepared figures and drafted the initial manuscript. G. R.: reviewed the manuscript. T.X., L.L., and Q.T.: revised and finalised the manuscript. All authors reviewed and approved the final manuscript.

\section{Author details}

${ }^{1}$ Chongqing Key Laboratory of Molecular Oncology and Epigenetics, The First Affiliated Hospital of Chongqing Medical University, Chongqing, China. 2Department of Oncology, The Second Affiliated Hospital of Chongqing Medical University, Chongqing, China. ${ }^{3}$ Cancer Epigenetics Laboratory, Department of Clinical Oncology, State Key Laboratory of Oncology in South China, Sir YK Pao Center for Cancer and Li Ka Shing Institute of Health Sciences, The Chinese University of Hong Kong and CUHK-Shenzhen Research Institute, Hong Kong, Hong Kong

\section{Ethics approval and consent to participate}

All participants provided written consent before enrolment. This study was approved by the Ethics Committees of the First Affiliated Hospital of Chongqing Medical University and conformed to the tenets of the Declaration of Helsinki.

\section{Consent for publication}

I confirm this manuscript is original. If accepted, the article will not be published elsewhere without the written consent of the publisher.

\section{Conflict of interest}

The authors declare that they have no conflict of interest.

\section{Publisher's note}

Springer Nature remains neutral with regard to jurisdictional claims in published maps and institutional affiliations.

Supplementary Information accompanies this paper at https://doi.org/ 10.1038/s41419-018-0604-z.

Received: 16 January 2018 Revised: 10 April 2018 Accepted: 17 April 2018 Published online: 14 May 2018

\section{References}

1. Lam, A. K. Molecular biology of esophageal squamous cell carcinoma. Crit. Rev. Oncol. Hematol. 33, 71-90 (2000).

2. Pennathur, A., Gibson, M. K., Jobe, B. A. \& Luketich, J. D. Oesophageal carcinoma. Lancet 381, 400-412 (2013).

3. Rustgi, A. K. \& El-Serag, H. B. Esophageal carcinoma. New Engl. J. Med. 371, 2499-2509 (2014).

4. Kaz, A. M. \& Grady, W. M. Epigenetic biomarkers in esophageal cancer. Cancer Lett. 342, 193-199 (2014).

5. Kamangar, F., Dores, G. M. \& Anderson, W. F. Patterns of cancer incidence, mortality, and prevalence across five continents: defining priorities to reduce cancer disparities in different geographic regions of the world. J. Clin. Oncol. 24, 2137-2150 (2006).

6. Zhang, M. et al. Methylation of DACT2 accelerates esophageal cancer development by activating Wnt signaling. Oncotarget 7, 17957-17969 (2016).

7. Chen, W. et al. Cancer statistics in China, 2015. CA: Cancer J. Clin. 66, 115-132 (2016).

8. Ma, K., Cao, B. \& Guo, M. The detective, prognostic, and predictive value of DNA methylation in human esophageal squamous cell carcinoma. Clin. Epigenetics. 8, 43 (2016).

9. Garcia-Rodriguez, L. A., Lagergren, J. \& Lindblad, M. Gastric acid suppression and risk of oesophageal and gastric adenocarcinoma: a nested case control study in the UK. Gut 55, 1538-1544 (2006).

10. Chen, X. X. et al. Genomic comparison of esophageal squamous cell carcinoma and its precursor lesions by multi-region whole-exome sequencing. Nat. Commun. 8, 524 (2017).
11. Castro, C., Peleteiro, B. \& Lunet, N. Modifiable factors and esophageal cancer: a systematic review of published meta-analyses. J. Gastroenterol. 53, 37-51 (2017).

12. Song, Q. et al. Chromosomal and genomic variations in esophageal squamous cell carcinoma: a review of technologies, applications, and prospections. J. Cancer 8, 2492-2500 (2017).

13. Alder, H., Taccioli, C., Chen, H., Jiang, Y. \& Smalley, K. J. Dysregulation of miR-31 and miR-21 induced by zinc deficiency promotes esophageal cancer. Carcinogenesis 33, 1736-1744 (2012).

14. Tanaka, K. et al. Frequent methylation-associated silencing of a candidate tumor-suppressor, CRABP1, in esophageal squamous-cell carcinoma. Oncogene 26, 6456-6468 (2007).

15. Shen, H. \& Laird, P. W. Interplay between the cancer genome and epigenome Cell 153, 38-55 (2013)

16. Toh, Y, Egashira, A. \& Yamamoto, M. Epigenetic alterations and their clinical implications in esophageal squamous cell carcinoma. Gen. Thorac. Cardiovasc. Surg. 61, 262-269 (2013)

17. Cheng, Y. et al. A novel 19q13 nucleolar zinc finger protein suppresses tumor cell growth through inhibiting ribosome biogenesis and inducing apoptosis but is frequently silenced in multiple carcinomas. Mol. Cancer Res. 10, 925-936 (2012).

18. Huntley, S. et al. A comprehensive catalog of human KRAB-associated zinc finger genes: insights into the evolutionary history of a large family of transcriptional repressors. Genome Res. 16, 669-677 (2006).

19. Margolin, J. F. et al. Kruppel-associated boxes are potent transcriptional repression domains. Proc. Natl Acad. Sci. USA 91, 4509-4513 (1994).

20. Sripathy, S. P., Stevens, J. \& Schultz, D. C. The KAP1 corepressor functions to coordinate the assembly of de novo HP1-demarcated microenvironments of heterochromatin required for KRAB zinc finger protein-mediated transcriptional repression. Mol. Cell. Biol. 26, 8623-8638 (2006).

21. Friedman, J. R. et al. KAP-1, a novel corepressor for the highly conserved KRAB repression domain. Genes. Dev. 10, 2067-2078 (1996).

22. Gebelein, B., Fernandez-Zapico, M., Imoto, M. \& Urrutia, R. KRAB-independent suppression of neoplastic cell growth by the novel zinc finger transcription factor KS1. J. Clin. Investig. 102, 1911-1919 (1998).

23. Gebelein, B. \& Urrutia, R. Sequence-specific transcriptional repression by KS1, a multiple-zinc-finger-Kruppel-associated box protein. Mol. Cell. Biol. 21, 928-939 (2001).

24. Cheng, Y. et al. KRAB zinc finger protein ZNF382 is a proapoptotic tumor suppressor that represses multiple oncogenes and is commonly silenced in multiple carcinomas. Cancer Res. 70, 6516-6526 (2010).

25. Sawada, G. et al. Genomic landscape of esophageal squamous cell carcinoma in a japanese population. Gastroenterology 150, 1171-1182 (2016).

26. Song, Y. et al. Identification of genomic alterations in oesophageal squamous cell cancer. Nature 509, 91-95 (2014).

27. Jones, P. A. Epigenetics in carcinogenesis and cancer prevention. Ann. N.Y. Acad. Sci. 983, 213-219 (2003).

28. Urrutia, R. KRAB-containing zinc-finger repressor proteins. Genome Biol. 4, 231 (2003).

29. Lupo, A. et al. KRAB-Zinc finger proteins: a repressor family displaying multiple biological functions. Curr. Genom. 14, 268-278 (2013).

30. Witzgall, R., O'Leary, E., Leaf, A., Onaldi, D. \& Bonventre, J. V. The Kruppelassociated box-A (KRAB-A) domain of zinc finger proteins mediates transcriptional repression. Proc. Natl. Acad. Sci. USA 91, 4514-4518 (1994).

31. Quenneville, S. et al. The KRAB-ZFP/KAP1 system contributes to the early embryonic establishment of site-specific DNA methylation patterns maintained during development. Cell Rep. 2, 766-773 (2012).

32. Wiznerowicz, M. et al. The Kruppel-associated box repressor domain can trigger de novo promoter methylation during mouse early embryogenesis. J. Biol. Chem. 282, 34535-34541 (2007).

33. Schmitz, G., Heimerl, S. \& Langmann, T. Zinc finger protein ZNF202 structure and function in transcriptional control of HDL metabolism. Curr. Opin. Lipidol. 15, 199-208 (2004)

34. Florio, F. et al. Biochemical and functional interaction between ZNF224 and ZNF255, two members of the Kruppel-like zinc-finger protein family and WT1 protein isoforms. Human. Mol. Genet. 19, 3544-3556 (2010).

35. Luo, K et al. Expression of a novel Krupple-like zinc-finger gene, ZNF382, in human heart. Biochem. Biophys. Res. Commun. 299, 606-612 (2002).

36. Luo, X. et al. The tumor suppressor interferon regulatory factor 8 inhibits betacatenin signaling in breast cancers, but is frequently silenced by promoter methylation. Oncotarget 8, 48875-48888 (2017). 
37. Xiang, T. et al. The ubiquitin peptidase UCHL1 induces G0/G1 cell cycle arrest and apoptosis through stabilizing p53 and is frequently silenced in breast cancer. PloS ONE 7, e29783 (2012).

38. Livak, K. J. \& Schmittgen, T. D. Analysis of relative gene expression data using real-time quantitative PCR and the 2(-Delta Delta $C(T))$ Method. Methods 25, 402-408 (2001).

39. Tao, Q. et al. Defective de novo methylation of viral and cellular DNA sequences in ICF syndrome cells. Human. Mol. Genet. 11, 2091-2102 (2002).

40. Tao, Q. et al. Methylation status of the Epstein-Barr virus major latent promoter C in iatrogenic B cell lymphoproliferative disease. Application of PCR-based analysis. Am. J. Pathol. 155, 619-625 (1999).

41. Mu, H. et al. Methylation of PLCD1 and adenovirus-mediated PLCD1 overexpression elicits a gene therapy effect on human breast cancer. Exp. Cell Res. 332, 179-189 (2015).

42. $\mathrm{Mu}$, J. et al. Dickkopf-related protein 2 induces $\mathrm{GO} / \mathrm{G} 1$ arrest and apoptosis through suppressing Wnt/beta-catenin signaling and is frequently methylated in breast cancer. Oncotarget 8, 39443-39459 (2017).

43. Langmead, B., Trapnell, C., Pop, M. \& Salzberg, S. L. Ultrafast and memoryefficient alignment of short DNA sequences to the human genome. Genome Biol. 10, R25 (2009).

44. Langmead, B. \& Salzberg, S. L. Fast gapped-read alignment with Bowtie 2. Nat Methods 9, 357-359 (2012).

45. Marioni, J. C., Mason, C. E., Mane, S. M., Stephens, M. \& Gilad, Y. RNA-seq: an assessment of technical reproducibility and comparison with gene expression arrays. Genome Res. 18, 1509-1517 (2008).

46. Trapnell, C., Pachter, L. \& Salzberg, S. L. TopHat: discovering splice junctions with RNA-Seq. Bioinformatics 25, 1105-1111 (2009).

47. Trapnell, $C$. et al. Differential gene and transcript expression analysis of RNAseq experiments with TopHat and Cufflinks. Nat. Protoc. 7, 562-578 (2012).
48. Kim, D. et al. TopHat2: accurate alignment of transcriptomes in the presence of insertions, deletions and gene fusions. Genome Biol. 14, R36 (2013).

49. Wang, L. et al. DEGseq: an R package for identifying differentially expressed genes from RNA-seq data. Bioinformatics 26, 136-138 (2010).

50. Anders, S. \& Huber, W. Differential expression of RNA-Seq data at the gene level-the DESeq package. Heidelberg, Germany: European Molecular Biology Laboratory (EMBL). (2012) http://watson.nci.nih.gov/bioc_mirror/packages/ 2.11/bioc/vignettes/DESeq/inst/doc/DESeq.pdf.

51. Yu, G., Wang, L. G. \& He, Q. Y. ChIPseeker: an R/Bioconductor package for ChIP peak annotation, comparison and visualization. Bioinformatics 31, 2382-2383 (2015).

52. Quinlan, A. R. \& Hall, I. M. BEDTools: a flexible suite of utilities for comparing genomic features. Bioinformatics 26, 841-842 (2010).

53. Thorvaldsdottir, H., Robinson, J. T. \& Mesirov, J. P. Integrative Genomics Viewer (IGV): high-performance genomics data visualization and exploration. Brief. Bioinforma. 14, 178-192 (2013).

54. Robinson, J. T. et al. Integrative genomics viewer. Nat. Biotechnol. 29, 24-26 (2011).

55. Young, M. D., Wakefield, M. J., Smyth, G. K. \& Oshlack, A. Gene ontology analysis for RNA-seq: accounting for selection bias. Genome Biol. 11, R14 (2010).

56. Kanehisa, M. et al. KEGG for linking genomes to life and the environment. Nucleic Acids Res. 36, D480-D484 (2008).

57. Dennis, G. et al. DAVID: Database for Annotation, Visualization, and Integrated Discovery. Genome biology. 4, P3 (2003).

58. Shannon, P. et al. Cytoscape: a software environment for integrated models of biomolecular interaction networks. Genome Res. 13, 2498-2504 (2003). 